\title{
Descriptive Summary of the Grande Ronde Basalt Type Section, Columbia River Basalt Group
}

V. E. Camp

S. M. Price

S. P. Reidel

October 1978

Prepared for the United States

Department of Energy

Under Contract EY-77-C-06-1030

Rockwell International

Rockwell Hanford Operations

Energy Systems Group

Richland, WA 99352 


\section{DISCLAIMER}

This report was prepared as an account of work sponsored by an agency of the United States Government. Neither the United States Government nor any agency Thereof, nor any of their employees, makes any warranty, express or implied, or assumes any legal liability or responsibility for the accuracy, completeness, or usefulness of any information, apparatus, product, or process disclosed, or represents that its use would not infringe privately owned rights. Reference herein to any specific commercial product, process, or service by trade name, trademark, manufacturer, or otherwise does not necessarily constitute or imply its endorsement, recommendation, or favoring by the United States Government or any agency thereof. The views and opinions of authors expressed herein do not necessarily state or reflect those of the United States Government or any agency thereof. 


\section{DISCLAIMER}

Portions of this document may be illegible in electronic image products. Images are produced from the best available original document. 


\title{
Q
}

\section{Rockwell International}

\author{
Rockwell Hanford Operations \\ Energy Systems Group \\ Richland, WA 99352
}

\author{
PREPARED FOR THE UNITED STATES DEPARTMENT OF ENERGY \\ UNDER CONTRACT EY-77-C-06-1030
}

\section{PRELIMINARY REPORT}

This Report contains information of a preliminary nature. It is subject to revision or correction and therefore does not represent a final Report. It was prepared primarily for internal use within The Rockwell Hanford Operations. Any expressed views and opinions are those of the Author and not necessarily of the Company.

NOTICE

This Report was prepared as an account of work sponsored by the United States Government. Neither the United States nor the United States Department of Energy, nor any of their Employees, nor any of their Contractors, Subcontractors, or their Employees, makes any warranty, express or implied, or assumes any legal liability or responsibility for the accuracy, completeness, or usefulness of any information, apparatus, product or process disclosed, or represents that its use would not infringe privately owned rights. 


\section{DESCRIPTIVE SUMMARY OP THE GRANDE RONDE BaSALT \\ TYPE SECTION, COLUMBIA RIVER BASALT GROUP}

V. E. Camp

Washington State University

S. M. Price

S. P. Reidel

Basalt Geosciences Unit Research Department Rockwe 11 Hanford Operations

for

Basalt Waste Isolation Program

October 1978

Rockwell International

Rockwel 1 Hanford Operations

Energy Systems Group

Richland, Washington 99352 


\section{A B S T R A C T}

The Grande Ronde Basalt type section, located in extreme southeasterm Washington, has been measured, sampled, and characterized. The section is 800 meters thick and is comprised of 35 Grande Ronde Basalt flows. These flows are divisible into 3 magnetostratigraphic units termed, in ascending order, the $R_{1}$, the $N_{1}$, and the $R_{2}$. The $R_{1}$ unit is represented by 13 reversely polarized flows; the $N_{1}$ unit by 13 normally polarized flows; and the $R_{2}$ by 9 reversely polarized flows. Chemically, the Grande Ronde Basalt flows have been divided into 2 major groups, termed $A$ and $B$. The compositions of the lower 9 flows, members of Group A, are similar to either the high-Mg Grande Ronde chemical-type, the high-Ti Grande Ronde chemical type, or the Pomona chemical type. The compositions of the upper 25 flows, members of Group B, are predominantly similar to the low-Mg Grande Ronde chemical type. Petrographically, the Grande Ronde Basalt flows are generally fine grained and aphyric and have an intergranular or intersertal micro-texture. Major mineral phases include plagioclase $\left(A n_{40-60}\right)$ and augite; minor mineral phases include pigeonite, orthopyroxene, ilmenite, titanomagnetite, and olivine. Croup A flows generally contain more olivine and less pigeonite than do Group $B$ flows. 
TABLE OF CONTENTS

Page

INTRODUCTION

GENERAL SETTING $\quad \cdot \quad 5$

DESCRIPTION OF THE GRANDE RONDE BASALT TYPE SECTION 9

FIELD PROPERTIES

CHEMISTRY

$\begin{array}{ll}\text { PETROGRAPHY } & 16\end{array}$

APPLICATION OF SECTION DATA 22

ACKNOWLEDGMENTS 22

BIBLIOGRAPHY 22

DISTRIBUTION

LIST OF FIGURES

FIGURE 1 COLUMBIA RIVER BASALT GROUP STRATIGRAPHIC NOMENCLATURE $\quad 6$

FIGURE 2 PHOTOMOSAIC OF THE GRANDE RONDE BASALT TYPE SECTION $\quad 7$

FIGURE 3 GEOLOGIC MAP OF THE SNAKE AND GRANDE RONDE RIVERS CONFLUENCE AREA

FIGURE 4 GRAPHIC REPRESENTATION OF THE GRANDE RONDE BASALT TYPE SECTION

FIGURE 5 LOCATION OF COLUMBIA RIVER BASALT DIKES OF THE GRANDE RONDE CHEMICAL TYPE IN SOUTHEASTERN WASHINGTON AND NORTHEASTERN OREGON

FIGURE 6 PHOTOMICROGRAPH OF ROGERSBURG FLOW AND TYPICAL APHYRIC GRANDE RONDE BASALT FLOW

\section{LIST OF TABLES}

TABLE I MAJOR OXIDE AVERAGE X-RAY FLUORESCENCE ANALYSES FOR FLOWS OF THE GRANDE RONDE BASALT TYPE SECTION 
List of Tables (continued)

Page

TABLE II TRACE ELEMENT AVERAGE X-RAY FLUORESCENCE ANALYSES FOR FLOWS OF THE GRANDE RONDE BASALT TYPE SECTION

TABLE III TRACE ELEMENT AVERAGE INSTRUMENTAL NEUTRON ACTIVATION ANALYSES FOR FLOWS OF THE GRANDE RONDE BASALT TYPE SECTION

TABLE IV MINERAL AND GLASS AVERAGE ELECTRON MICROPROBE ANALYSES FOR FLOWS OF THE GRANDE RONDE BASALT TYPE SECTION

TABLE $V$

MODAL ANALYSES FOR SAMPLES FROM THE GRANDE RONDE BASALT TYPE SECTION

TABLE VI PETROGRAPHY OF BASALT SAMPLES FROM THE GRANDE RONDE BASALT TYPE SECTION 


\section{INTRODUCTION}

A formal revision of the Columbia River Basalt Group stratigraphic nomenclature established by Waters (1961) has recently been outlined by Swanson, et al., (1978). Under this new stratigraphic framework (Figure 1), the Yakima Basalt is raised from formation to subgroup status, and three formations (the Grande Ronde Basalt, Wanapum Basalt, and Saddle Mountains Basalt, in order of decreasing age) are defined within it. The type section for the Grande Ronde Basalt is designated as a sequence of flows comprising a prominent west-trending spur ridge located near the confluence of the Grande Ronde and Snake rivers, southeast Washington (Figure 2) (Swanson, et al., 1978). This section lies at the intersection of three doctoral dissertation areas and has been sampled jointly and described separately by Camp (1976), Price (1977), and Reidel

(1978). In order to provide one central reference publication, a summary of the section data generated by these individuals is presented in this document.

\section{GENERAL SETTING}

The type section for the Grande Ronde Basalt is designated as the "prominent west-trending spur ridge extending from the northwest quarter of Section 23 across the north third of Section 22 to the northeast quarter of Section 21, Township 7 North, Range 46 East., Black Butte quadrangle, in the lower part of the Grande Ronde River valley, Asotin County, extreme southeast Washington" (Figure 3) (Swanson, et al., 1978). The section is situated near the eastern margin of the Columbia Plateau physiographic province within the canyon country of the middle Snake River and major tributaries. Lower Grande Ronde Canyon exposures of the Columbia River Basalt sequence are over 1,000 meters thick and are comprised of flows of the Imnaha Basalt, Grande Ronde Basalt, Wanapum Basalt, and Saddle Mountains Basalt (Figure 1) and dikes of the Chief Joseph swarm (Figure 3) (Gibson, 1969; Taubeneck, 1970; and, Price, 1977). Just to the east of the section locality, the base of the Imnaha 


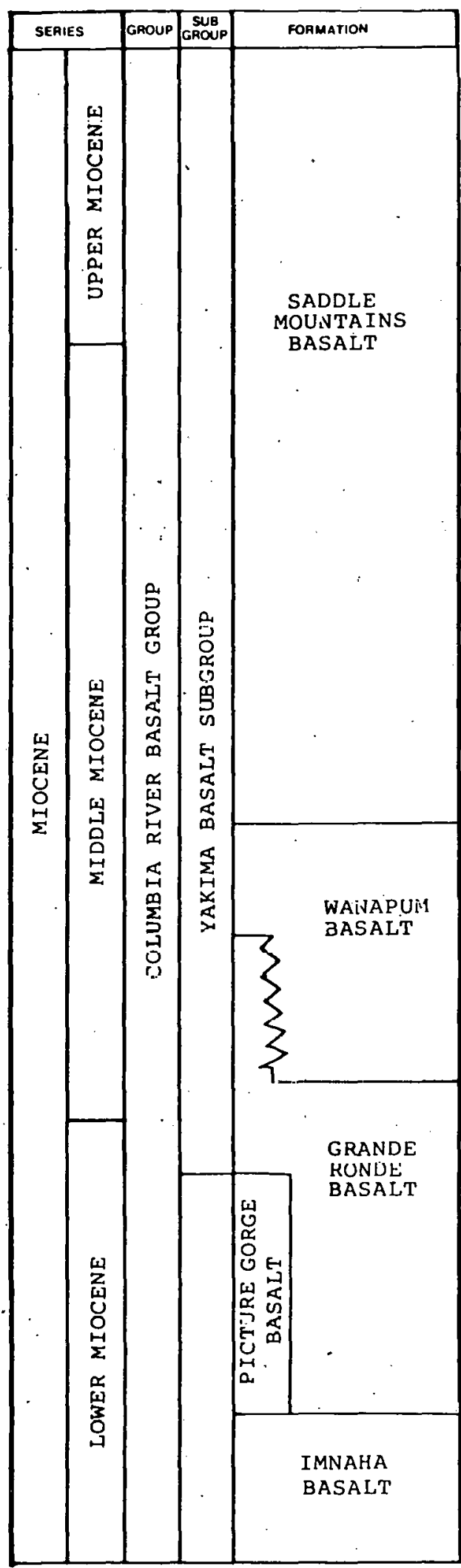

FIGURE 1 


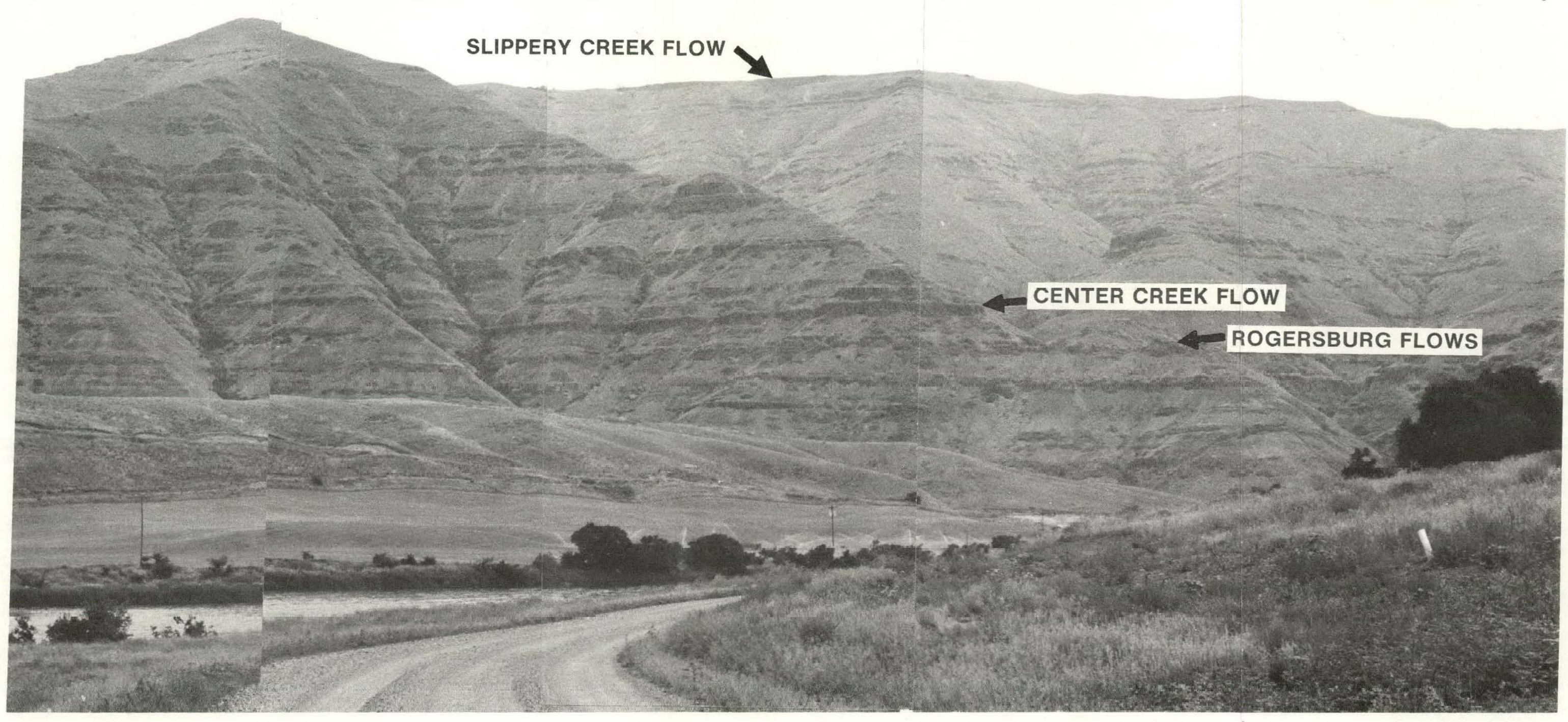

FIGURE 2

PHOTOMOSAIC OF THE GRANDE RONDE BASALT TYPE SECTION 


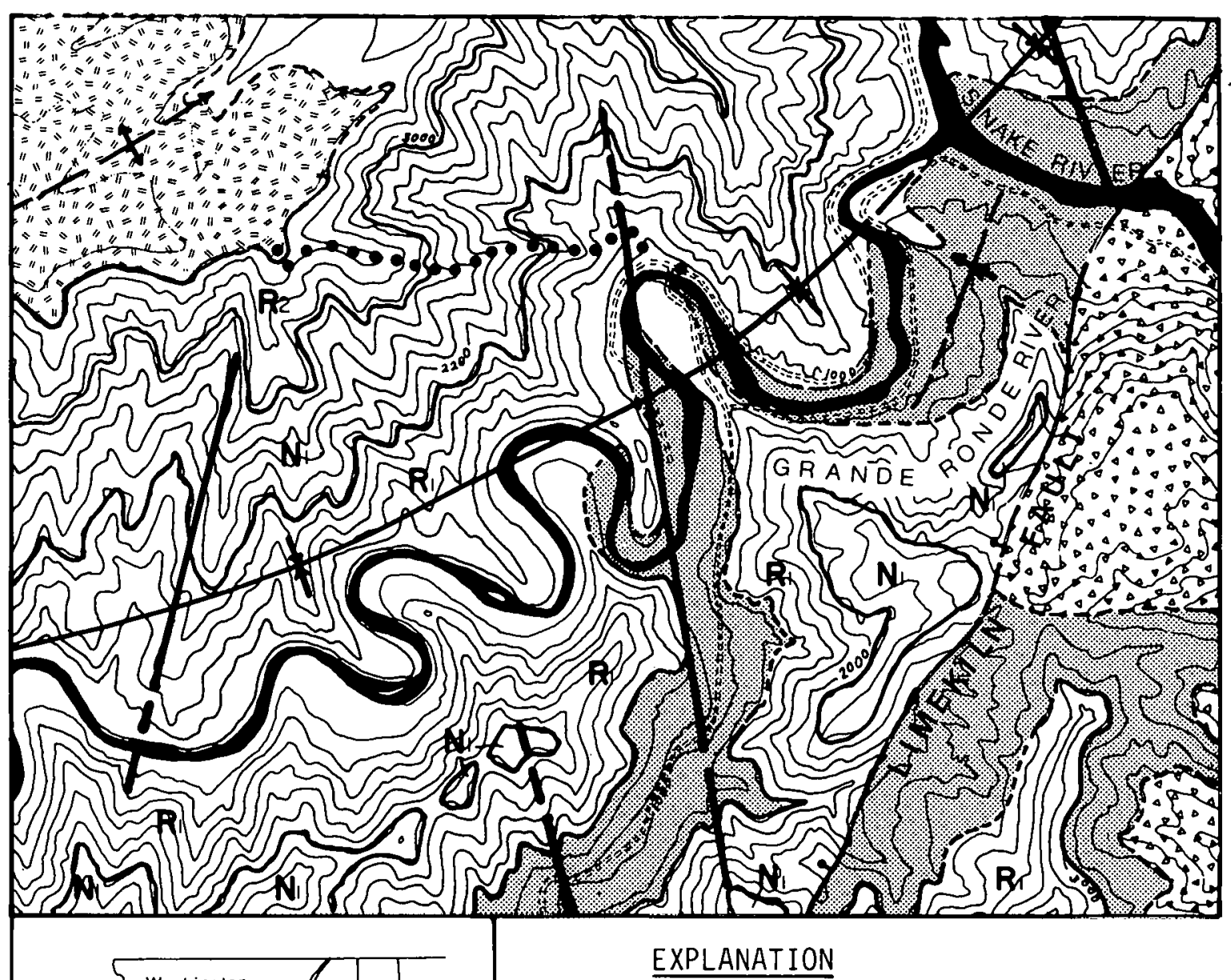

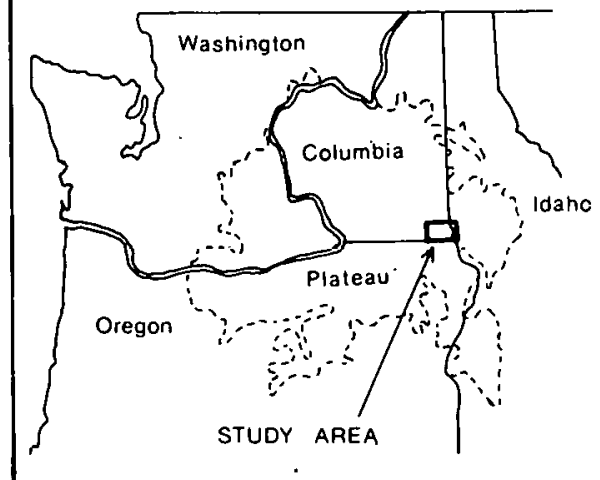
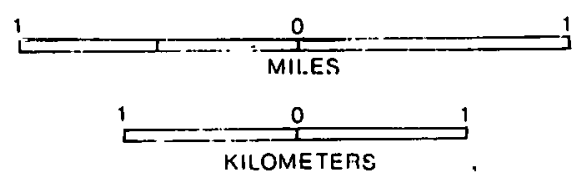

Contour Interval is 200 Feet
Fadd Salt

$\square$ Grande Ronde Basalt

Imnaha Basalt

$\because$ Metamorphic Complex

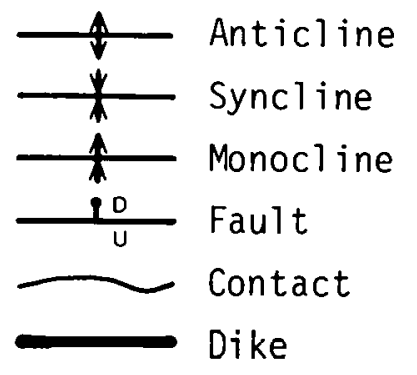

- - Grande Ronde Section

FIGURE 3

GEOLOGIC MAP OF THE SNAKE AND GRANDE RONDE RIVERS CONFLUENCE AREA 
Basalt rests unconformably on pre-Tertiary metamorphic rocks (Figure 3 ). Structurally, the section lies near the eastern terminus of the Blue Mountain uplift and just to the north of the Limekiln fault (Figure 3) (Camp, 1976; Price, 1977; and, Reidel, 1978).

\section{DESCRIPTION OF THE GRANDE RONDE BASALT TYPE SECTION}

\section{FIELD PROPERTIES}

During section measurement, elevations of flow and interbed contacts were recorded using a surveying altimeter calibrated at two-foot intervals. In general, section samples were collected from the bottom, center, and top of each definable flow. Fol lowing sampling, flow outcrop characteristics, hand specimen petrography, and remanent magnetization measurements were recorded. Remanent magnetization measurements were made using a portable flux gate magnetometer.

A graphic representation of the Grande Ronde measured section, including the position of the 61 sample localities, is shown in Figure 4. The section is comprised of an 800-meter thick sequence of 35 Grande Ronde Basalt flows overlain by an interbed and a single 50-meter thick Saddle Mountains Basalt flow. The lower portion of the section is cross-cut by one Grande Ronde Basalt dike (Price, 1977). Just to the east of the section locality, the base of the Grande Ronde Basalt is exposed and overlies the Imnaha Basalt with no apparent unconformity.

The Grande Ronde Basalt flows of the section are predominantly cliff-formers, and are responsible for the stair-step character of the walls of the lower Grande Ronde Canyon (Figure 2). The internal structures of individual flows are variable, ranging from those displaying well-developed colonnades and entablatures to those displaying no such recognizable subdivisions. The Grande Ronde Basalt flows of the section range in thickness from 7 to 70 meters, with an average flow thickness of 16 meters. 


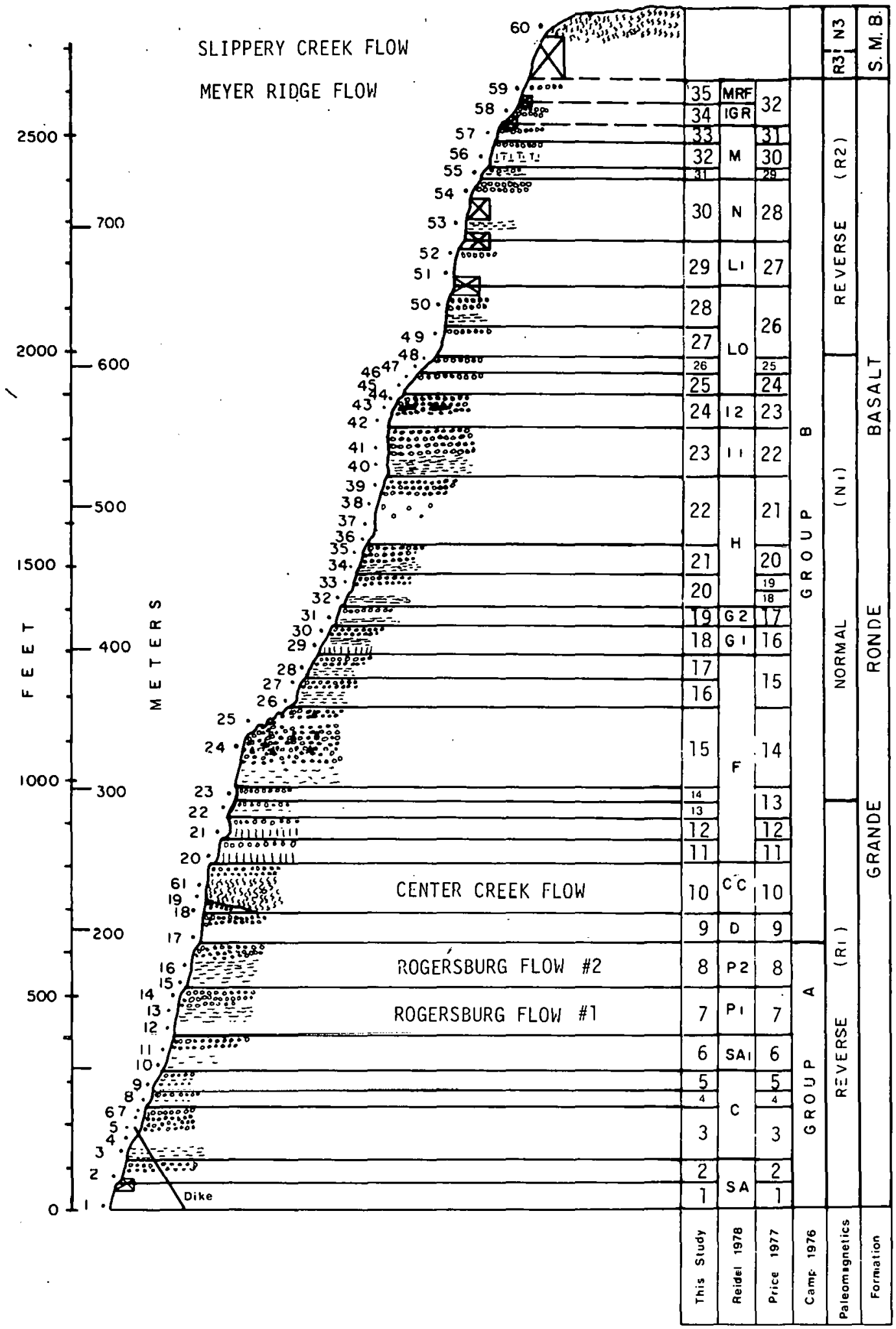

FIGURE 4

GRAPHIC REPRESENTATION OF THE GRANDE RONDE BASALT TYPE SECTION 
Three magnetostratigraphic units are definable within the Grande Ronde Basalt sequence at the type locality (Figure 4). Following the terminology suggested by Swanson and Wright (1976), these units are termed, in ascending order, the $R_{1}, N_{1}$, and $R_{2}$. The $R_{1}$ is represented by a 300 -meter thick sequence of 13 reversely polarized flows; the $N_{1}$ by a 300 -meter thick sequence of 13 normally polarized flows; and, the $R_{2}$ by a 250-meter thick sequence of 9 normally polarized flows. Flows of the youngest Grande Ronde Basalt magnetostratigraphic unit, the $\mathrm{N}_{2}$ (Swanson and Wright, 1976), are not represented within the section. The single Saddle Mountains Basalt flow capping the section has a normal polarity. Named flows within the section include the Rogersburg flows (Price, 1977) the Center Creek flow (Bond, 1962; Reidel, 1978), the Meyer Ridge flow (Camp, 1976) of the Grande Ronde Basalt, and the Slippery Creek flow (Price, 1977) (the Uniontown-3 flow of Camp, 1976) of the Saddle Mountains Basalt (Figure 4). The Rogersburg flows (the porphyritic flows of Camp, 1976; Reidel, 1978) are part of a distinctive plagioclase-phyric sequence present throughout the canyon country of the middle Snake River and major tributaries (Gibson, 1969; Breeser, 1972; Camp, 1976; Price, 1977; Reidel, 1978; and Ross, 1978). As such, the flow sequence is an important "regional" stratigraphic marker unit. Recognition of the other three named flows is predominantly based on field position and chemical criteria. The areal extent and distinguishing properties of the Rogersburg, Center Creek, Meyer Ridge, and Sippery Creek flows are discussed in detail in the authors' dissertations (Camp, 1976; Price, 1977; and, Reide1, 1978).

\section{CHEMISTRY}

The 61 samples collected from the Grande Ronde Basalt type section were analyzed by Reidel for $\mathrm{SiO}_{2}, \mathrm{Al}_{2} \mathrm{O}_{3}, \mathrm{TiO}_{2}$, total Fe-oxides as $\mathrm{FeO}, \mathrm{MgO}, \mathrm{CaO}, \mathrm{Na}_{2} \mathrm{O}, \mathrm{K}_{2} \mathrm{O}, \mathrm{MnO}$, and $\mathrm{P}_{2} \mathrm{O}_{5}$, using Washington State University's wave length dispersive $X$-ray fluorescence spectrometer. The analytical method employed is described in Hooper, et al., (1976). The $X$-ray fluorescence analytical results are reported in Camp (1976) and Reidel (1978). Splits of the 61 section samples were also analyzed for the 
same elements using atomic absorption spectroscopy and color spectrophotometry; these results are reported in Price (1977). Because comparable chemical trends were obtained using both analytical methods, only the $\mathrm{X}$-ray fluorescence analytical results are included in this paper. The average major oxide compositions of the 35 Grande Ronde Basalt flows (as defined in Figure 4) computed from the $X$-ray fluorescence analytical data base are reported in Table $I$.

Analytical values for selected trace elements ( $\mathrm{Zr}, \mathrm{Sr}, \mathrm{Rb}, \mathrm{Ba}, \mathrm{V}, \mathrm{Sc}$, $\mathrm{Ni}, \mathrm{Cr}, \mathrm{Cu}$, and $Y$ ) using the $X$-ray fluorescence analytical technique were determined by Reidel (1978). Analyses for additional trace elements (La, $\mathrm{Ce}, \mathrm{Sm}, \mathrm{Eu}, \mathrm{Tb}, \mathrm{Yb}, \mathrm{Lu}, \mathrm{Co}, \mathrm{Cr}, \mathrm{Hf}, \mathrm{Th}, \mathrm{Ta}$, and $\mathrm{Cs}$ ) using instrumental neutron activation analys is were obtained by Price (1977) and Reidel (1978). Available trace element data are listed in Tables II and III. An examination of major, minor, and selected trace element analyses for flows comprising the Grande Ronde Basalt type section showed that a chemical break occurs in the vicinity of the Center Creek flow. Camp (1976) termed flows below this chemical break members of Group A and flows above this break members of Group B. This same terminology was also applied by Price (1977) and Reidel (1978). However, Reidel (1978) added one more flow to the Group $A$ sequence (Figure 4).

In general, the compositions of the 9 Group A flows are similar to either the high-Mg Grande Ronde, the high-Ti Grande Ronde, or the Pomona chemical types of Wright, et al. (1973) and Swanson and Wright (1978). With the exception of the Meyer Ridge flow, the chemistry of the 29 Group $B$ flows resembles their low-Mg Grande Ronde chemical type. The composition of the Meyer Ridge flow resembles their high-Mg Grande Ronde chemical type or their Pomona chemical type. In general, Group A flows have a lower $\mathrm{SiO}_{2}$ (average difference of 2 percent) and a higher MgO (average difference of 0.7 percent) content than do Group B Grande Ronde Basalt flows.

The flows of the Grande Ronde section were further divided on the basis of chemistry by Reidel (1978). This breakdown, based on a more detailed evaluation of observed chemical variation, is noted in figure 4. Discriminant analysis of these flow categories by Reidel (1978) showed that they have a statistical validity based on the chemical analyses. 
TABLE I

MAJOR OXIDE AVERAGE X-RAY FLUORESCENCE ANALYSES FOR FLOWS OF THE GRANDE RONDE BASALT TYPE SECTION' ${ }^{1}$

\begin{tabular}{|c|c|c|c|c|c|c|c|c|c|c|c|}
\hline $\mathrm{FLOW}^{2}$ & $\mathrm{SiO}_{2}$ & $\mathrm{Al}_{2} \mathrm{O}_{3}$ & $\mathrm{TiO}_{2}$ & $\mathrm{FeO}^{3}$ & MnO & $\mathrm{CaO}$ & $\mathrm{MgO}$ & $k_{2} O$ & $\mathrm{Na}_{2} \mathrm{O}$ & $\mathrm{P}_{2} \mathrm{O}_{5}$ & $\begin{array}{c}\text { SAMPLE } \\
\text { ANALYSES }^{2} \\
\end{array}$ \\
\hline$\overline{S M-1^{4}}$ & 50.17 & 14.31 & 2.25 & 11.02 & 0.21 & 10.04 & 5.33 & 0.94 & 3.03 & 0.42 & 60 \\
\hline $35^{5}$ & 52.83 & 14.91 & 1.61 & 8.46 & 0.19 & 10.16 & 5.99 & 0.95 & 2.64 & 0.26 & $\dot{59}$ \\
\hline 34. & 53.33 & 15.05 & 1.80 & 10.51 & 0.22 & 8.62 & 4.17 & 1.21 & 2.81 & 0.28 & 58 \\
\hline 33 & 54.76 & 14.97 & 2.47 & 9.35 & 0.21 & 7.61 & 3.58 & 1.91 & 2.74 & 0.41 & 57 \\
\hline 32 & 53.05 & 14.29 & 2.22 & 11.25 & 0.21 & 8.00 & 4.01 & 1.82 & 2.77 & 0.38 & 56 \\
\hline 31 & 52.79 & 14.30 & 2.25 & 11.70 & 0.21 & 7.88 & 4.06 & 1.72 & 2.72 & 0.37 & 55 \\
\hline 30 & 53.50 & 14.72 & 2.51 & 11.21 & 0.21 & 7.34 & 3.52 & 1.94 & 2.63 & 0.39 & 53,54 \\
\hline 29 & 55.55 & 14.48 & 2.35 & 11.15 & 0.20 & 6.93 & 3.23 & 2.02 & 2.68 & 0.47 & $51: 52$ \\
\hline 28 & 55.04 & 14.44 & 2.22 & 10.64 & 0.19 & 6.98 & 3.40 & 1.04 & 2.79 & 0.36 & 50 \\
\hline 27 & 54.45 & 14.48 & 2.33 & 10.27 & 0.23 & 7.25 & 3.31 & 1.97 & 3.34 & 0.36 & 49 \\
\hline 26 & 53.02 & 14.52 & 2.28 & 11.11 & 0.21 & 8.16 & 4.12 & 1.43 & 2.82 & 0.35 & 47,48 \\
\hline 25 & 52.95 & 14.64 & 2.36 & 11.41 & 0.21 & 7.92 & 3.82 & $1: 39$ & 2.92 & 0.41 & 45,46 \\
\hline 24 & 54.26 & 15.17 & 1.81 & 9.34 & 0.20 & 8.52 & 1.61 & 1.19 & 2.62 & 0.27 & $42,43,44$ \\
\hline 23 & 53.73 & 14.79 & 1.90 & 10.61 & 0.21 & 7.94 & 4.32 & 1.39 & 2.83 & 0.31 & 40,41 \\
\hline 22 & 52.98 & 14.68 & $2.10^{\circ}$ & 11.39 & 0.22 & 8.14 & 4.13 & 1.33 & 2.69 & 0.35 & $36,37,38,38$ \\
\hline 21 & 53.39 & 14.36 & 2.24 & 11.44 & 0.20 & 7.88 & 3.82 & 1.62 & 2.71 & 0.38 & 34,35 \\
\hline 20 & 53.78 & 14.85 & 1.97 & 10.28 & 0.22 & 8.39 & 4.46 & 1.36 & 2.37 & 0.33 & 32,33 \\
\hline 19 & 53.08 & 15.07 & 1.78 & 9.66 & 0.21 & 9.18 & 3.14 & 1.19 & 2.43 & 0.26 & 31 \\
\hline 18 & 55.64 & 15.06 & 1.70 & 9.13 & 0.19 & 7.88 & 4.29 & 1.50 & 2.39 & 0.23 & 29,30 \\
\hline 17 & 54.46 & 14.38 & 2.08 & 11.12 & 0.20 & 7.55 & 3.79 & 1.69 & 2.43 & 0.30 & 28 \\
\hline 16 & 54.56 & 14.81 & $2.1 ?$ & 10.47 & $0.20^{\circ}$ & 7.44 & 3.92 & $\cdot 1.57$ & 2.65 & 0.38 & 26,27 \\
\hline 16 & 61.81 & 14.81 & 2.00 & 10.J1 & 0.19 & 7.01 & 4.02 & 1.58 & 2.32 & 0.28 & $24 ;, \angle b$ \\
\hline 14 & 56.23 & 14.79 & 2.17 & 9.96 & 0.19 & 6.67 & 3.05 & 1.98 & 2.68 & 0.28 & 23 \\
\hline 13 & 55.99 & 14.62 & 2.20 & 10.17 & 0.20 & $6.7 ?$ & 3.14 & 2.22 & 2.46 & 0.28 & $? ?$ \\
\hline 12 & 54.07 & 14.48 & 2.14 & 10.61 & 0.20 & 7.89 & 4.18 & 1.57 & 2.55 & ก 3? & 21 \\
\hline 11 & 54.37 & 14.20 & 2.34 & 10.89 & 0.22 & 7.37 & 3.51 & $1.9 n$ & 2.79 & $n$ an & ?ח \\
\hline $10^{6}$ & 54.10 & 14.62 & 2.21 & 10.51 & 0.22 & 7.58 & 3.99 & 1.70 & 2.71 & 0.37 & $18,19,61$ \\
\hline $9^{7}$ & 53.51 & 14.62 & 2.15 & 10.18 & 0.19 & 8.39 & 4.64 & 1.46 & 2.56 & 0.30 & 17 \\
\hline $8^{7}$ & 53.25 & 14.68 & 2.22 & 10.07 & 0.20 & 8.61 & 4.79 & 1.35 & 2.55 & 0.30 & 15,16 \\
\hline 7 & 53.19 & 14.59 & 2.35 & 10.43 & 0.22 & 8.37 & 4.41 & 1.42 & 2.66 & 0.36 & $12,13,14$ \\
\hline 6 & 53.06 & 14.50 & 2.30 & 11.12 & 0.25 & 8.10 & 4.17 & 1.41 & 2.77 & 0.35 & 10,11 \\
\hline 5 & 53.37 & 15.18 & 1.90 & 9.71 & 0.22 & 9.60 & 5.25 & $0: 91$ & 2.58 & 0.27 & 9 \\
\hline 4 & פ1.31 & 15.01 & $2.32^{\circ}$ & 10.70 & 0.23 & 9.41 & 5.29 & 0.90 & 2.45 & 0.30 & 8 \\
\hline 3 & 52.11 & 14.92 & 2.42 & 10.25 & 0.21 & 9.16 & 5.00 & 1.17 & 2.46 & 0.33 & $3,4,6,7$ \\
\hline 2 & 52.80 & 14.84 & 2.17 & 10.81 & 0.22 & 8.54 & 4.48 & 0.97 & 2.81 & 0.37 & 2 \\
\hline 1.8 & 52.84 & 14.74 & 2.31 & 11.27 & 0.23 & 7.91 & 4.43 & 1.18 & 2.67 & 0.38 & 1 \\
\hline Dike & 53.44 & 14.67 & 2.17 & 11.40 & 0.20 & 7.84 & 3.91 & 1.68 & 2.33 & 0.35 & 5 \\
\hline
\end{tabular}

\footnotetext{
${ }^{1}$ Average weight percent compositions computed from XRF analyses reported in Reidel (1978):

${ }^{2}$ Sce Figure 4 for flow divisions and salliple lucalities.

${ }^{3} \mathrm{Fe}_{2} \mathrm{O}_{3}$ is expressed as a constant 2.00 weight percent.

4 Uniontown-3 flow of Camp (1976), Slippery Creek flow of Price (1977); Saddle Mountains Basalt flow.

${ }^{5}$ Meyer Ridge of Camp (1976); Grande Ronde Basalt flow.

${ }^{6}$ Center Creek flow of Reidel (1978).

7 Rogersburg flows of Price (1977) and porphyritic flows of Camp (1976) and Reidel (1978).

8 Dike location noted in Figure 4.
} 


\section{TABLE II}

TRACE ELEMENT AVERAGE X-RAY FLUORESCENCE ANALYSES FOR FLOWS OF THE GRAINDE RONDE BASALT TYPE SECTION 1

\begin{tabular}{|c|c|c|c|c|c|c|c|c|c|c|c|}
\hline FLOW2 & $\mathrm{Zr}$ & $s r$ & $\mathrm{Rb}$ & $\mathrm{Ba}$ & v & Sc & $\mathrm{Ni}$ & $\mathrm{Cr}$ & $\mathrm{Cu}$ & $\gamma$ & $\begin{array}{c}\text { SAMPLE } \\
\text { ANALYSES }^{2}\end{array}$ \\
\hline $\operatorname{sit}-1^{3}$ & 179 & 233 & 27 & 368. & 333 & 33 & 43 & 30 & 244 & 10 & 60 \\
\hline $35^{4}$ & 163 & 347 & 35 & 350 & 308 & 45 & 118 & 145 & 173 & 8 & 59 \\
\hline 34 & 174 & 340 & 36 & 569 & 282 & 40. & 67 & 70 & 195 & 15 & 58 \\
\hline 33 & 220 & 390 & 50 & 1000 & 375 & 34 & 46 & 2 & 128 & 39 & 57 \\
\hline 32 & 195 & 207 & 47 & 616 & 348 & 38 & 33 & 10 & 105 & 14 & 56 \\
\hline 31 & 195 & 317 & 44 & 661 & 406 & 40 & 29 & 11 & 185 & 14 & 55 \\
\hline 30 & 229 & 329 & 45 & 748 & 387 & 34 & 63 & 20 & 92 & 14 & 53,54 \\
\hline 29 & 232 & 322 & 51 & 757 & 357 & 33 & 45 & 4 & 12 & 21 & 51,52 \\
\hline 28 & 213 & 346 & 47 & 675 & 342 & 32 & 89 & 10 & 75 & 17 & 50 \\
\hline 27 & 177 & 373 & 54 & 866 & 355 & 35 & 84 & 12 & 1 & 12 & 49 \\
\hline 26 & 197 & 336 & 38 & 560 & 395 & 40 & 39 & 34 & 17 & 13 & 47,48 \\
\hline 25 & 187 & 342 & 39 & 577 & 389 & 39 & 66 & 33 & 29 & 23 & 45,46 \\
\hline 24 & 190 & 355 & 36 & 469 & 341 & 40 & 32 & 37. & 63 & 14 & $42,43,44$ \\
\hline 23 & 173 & 325 & 43 & 517 & 366 & 40 & 34 & 32 & 50 & 20 & 40,41 \\
\hline 22 & 181 & 326 & 39 & 543 & 345 & 39 & 44 & 41 & 83 & 16 & $36,37,38,39$ \\
\hline 21 & 179 & 333 & 40 & 547 & 389 & 39 & 57 & 40 & 50 & 17 & 34,35 \\
\hline 20 & 186 & 336 & 36 & 520 & 352 & 39 & 39 & 1.9 & 54 & 9 & 32,33 \\
\hline 19 & 178 & 362 & 37 & 465 & 296 & 39 & 23 & 36 & .1 & 9 & 31 \\
\hline 18 & 190 & 340 & 42 & 495. & 339 & 36 & 49 & 14 & 57 & 22 & 29,30 \\
\hline 17 & 212 & 313 & 46 & 600 & 411 & 36 & 54 & 18 & 39 & 15 & 28 \\
\hline 16 & 210 & 334 & 40 & 686 & 410 & 39 & 46 & 17 & 63 & 15 & 26,27 \\
\hline 15 & 208 & 322 & 44 & 565 & 341 & 35 & 33 & 15 & 485 & 9 & 24,25 \\
\hline 14 & 240 & 345 & 45 & 706 & 405 & 33 & 48 & 3 & 111 & 12 & 23 \\
\hline 13 & 240 & 331 & 51 & 751 & 417 & 30 & 43 & 14 & 92 & 29 & 22 \\
\hline 12 & 209 & 320 & 41 & 603 & 426 & 37 & 45 & 34 & 23 & 21 & 21 \\
\hline 11 & 257 & 315 & 42 & 655 & 380 & 36 & 41 & 36 & 137 & 28 & 20 \\
\hline $10^{5}$ & 226 & 323 & 34 & 556 & 360 & 35 & 46 & 23 & 157 & 12 & $18,19,61$ \\
\hline $9^{6}$ & 211 & 354 & 44 & 495 & 395 & 35 & 119 & 49 & 178 & 37 & 17 \\
\hline $8^{6}$ & 217 & 353 & 38 & 494 & 370 & 35 & 62 & 58 & 205 & 7 & 15,16 \\
\hline 7 & 218 & 356 & 40 & 627 & 391 & 36 & 61 & 43 & 170 & 11 & $12, .13,14$ \\
\hline 6 & 207 & 319 & 39 & 442 & 383 & 39 & 28 & 21 & 174 & 18 & 10,11 \\
\hline 5 & 183 & 362 & 39 & 362 & 334 & 39 & 56 & 86 & 223 & 13 & 9 \\
\hline .4 & 189 & 340 & 30 & 391 & 335 & 37 & 57 & 75 & 65 & 10 & 8 \\
\hline 3 & 205 & 318 & 37 & 521 & 379 & 38 & $8 ?$ & 88 & 118 & 12 & $3,4,6,7$ \\
\hline 2 & 205 & 333 & 30 & 501 & 435 & 44 & 43 & 28 & 19.1 & 26 & 2 \\
\hline 1 & 232 & 303 & 10 & 499 & 170 & 42 & 57 & 42 & 125 & 25 & 1 \\
\hline Dike $^{7}$ & 189 & 333 & 49 & 495 & 467 & 37 & 40 & 38 & 113 & 32 & \\
\hline
\end{tabular}

${ }^{1}$ Average value in parts per million computed from XRF analyses reported in Reidel (1978).

2 See Figure 4 for flow divisions and sample localities.

3 Uniontown-3 flow of Camp (1976), Slippery Creek flow of Price (1977); Saddle Mountains Bâsalt tlow.

${ }^{4}$ Meyer Ridge flow of Camp (1976); Grande Ronde Basalt flow.

${ }^{5}$ Center Creek flow of Reidel (1978).

${ }^{6}$ Rogersburg flows of Price (1977) and porphyritic flows of Camp (1976) and Reidel (1978).

${ }^{7}$ Dike location. noted in Figure 4. 
TABLE III

-TRACE ELEMENT AVERAGE INSTRUMENTAL NEUTRON ACTIVATION ANALYSES FOR FLOWS OF THE GRANDE RONDE BASALT TYPE SECTIONT

\begin{tabular}{|c|c|c|c|c|c|c|c|c|c|c|c|c|c|c|}
\hline FLOW ${ }^{2}$ & La & $\mathrm{Ce}$ & Sm & $\mathrm{Eu}$ & Tb & $Y b$ & Lu & Co & $\mathrm{Cr}$ & $\mathrm{Hf}$ & Th & $\mathrm{Ta}$ & Cs & $\begin{array}{c}\text { SAMPLE } \\
\text { ANALYSES } \\
\end{array}$ \\
\hline$S M-1^{3}$ & 29.7 & ND & 6.7 & 2.1 & 1.2 & 4.7 & ND & 44 & 45.4 & 4.5 & 4.6 & 2.0 & ND & 60 \\
\hline $35^{4}$ & 18.2 & 24.7 & 4.5 & 1.5 & 0.8 & 3.6 & ND & 36 & 141.0 & 3.5 & 3.4 & 1.5 & 0.5 & 59 \\
\hline 34 & 25.1 & 29.8 & 6.3 & 1.9 & 1.0 & ND & ND & ND & 75.4 & 3.6 & 3.4 & 1.2 & ND & 58 \\
\hline 33 & 29.1 & 59.4 & 6.9 & 2.1 & 1.0 & 5.2 & ND & 44 & 8.6 & 5.3 & 5.9 & 1.4 & 1.0 & 57 \\
\hline 32 & 26.7 & 40.2 & 7.8 & 2.2 & ND & ND & ND & ND & 16.8 & 3.0 & 4.3 & 1.9 & 1.6 & 56 \\
\hline 31 & 26.3 & 38.2 & 7.2 & 2.1 & ND & ND & ND & ND & 16.5 & 4.9 & 5.1 & 2.0 & 1.1 & 55 \\
\hline 30 & 25.6 & 44.6 & 6.8 & 1.9 & ND & ND & 0.61 & 34 & 13.7 & 5.0 & 5.1 & 1.4 & 1.6 & 53,54 \\
\hline 29 & 30.1 & 44.6 & 6.9 & 2.1 & 1.0 & 4.1 & ND & 28 & 10.0 & 5.6 & 5.2 & 1.4 & 1.5 & 51 \\
\hline 28 & 26.3 & 45.3 & 6.2 & 2.3 & 1.2 & ND & ND & ND & 6.1 & 5.2 & 7.5 & 1.8 & 1.5 & 50 \\
\hline 27 & ND & ND & ND & ND & ND & ND & ND & ND & ND & ND & ND & ND & ND & -- \\
\hline 26 & 22.8 & $N D$ & 6.6 & 2.0 & 1.0 & 4.7 & ND & 45 & 17.3 & 4.6 & 4.7 & 1.6 & ND & 47,48 \\
\hline 25 & 25.2 & 63.9 & 7.2 & 2.4 & ND & ND & ND & ND & 22.4 & 4.3 & 4.8 & 1.5 & 1.0 & 46 \\
\hline 24 & 22.9 & 52.7 & 5.8 & 1.9 & 1.0 & ND & ND & ND & 19.5 & 4.5 & 3.7 & 1.6 & 1.1 & 43 \\
\hline 23 & 20.0 & 49.2 & 4.9 & 1.8 & 0.8 & 5.6 & ND & 33 & 32.3 & 4.5 & 3.6 & 1.3 & ND & 40,41 \\
\hline 22 & 21.2 & 50.5 & 6.2 & 2.0 & ND & Nō & Nंธ & ND & 41.6 & 4.2 & 3.9 & 1.2 & 0.6 & 31 \\
\hline 21 & 23.0 & 51.9 & 6.5 & 2.1 & 1.2 & ND & ND & ND & 29.1 & 3.7 & 4.2 & 1.0 & ND & 35 \\
\hline 20 & 18.5 & 45.4 & 5.1 & 1.8 & 1.2 & ND & ND & ND & ND & 3.4 & 3.3 & 1.5 & 0.4 & 32 \\
\hline 19 & 20.7 & 42.6 & 5.3 & 1.6 & 1.0 & 3.2 & ND & 36 & 38 & 4.4 & 4.0 & 1.2 & ND & 31 \\
\hline 18 & 20.8 & 47.5 & 5.6 & 1.8 & 0.8 & ND & ND & ND & ND & 3.7 & 4.3 & 1.9 & ND & 29 \\
\hline 17 & 20.1 & 50.2 & 5.3 & 2.0 & 1.1 & ND & ND & ND & ND & 4.3 & 3.8 & 1.6 & ND & 28 \\
\hline 16 & ND & 62.5 & ND & 2.2 & ND & ND & ND & ND & ND & 5.1 & 3.5 & 1.7 & ND & 26 \\
\hline 15 & 21.3 & 51.3 & 5.5 & 1.9 & 1.0 & 3.5 & ND & 35 & 14 & 4.8 & 5.1 & 1.4 & $\mathrm{NU}$ & 26 \\
\hline 14 & 23 & ND & 6.4 & 2.0 & ND & ND & ND & 33 & ND & 5.7 & 6.1 & ND & ND & 23 \\
\hline 13 & ND & ND & ND & ND & ND & ND & ND & ND & ND & ND & ND & ND & ND & -- \\
\hline 12 & 29.1 & 58.6 & 7.2 & 2.0 & ND & ND & ND & ND & 47.8 & 49 & 4.4 & 1.8 & ND & 21 \\
\hline וו & $9 r_{1}, r_{11}$ & 5 & 6.8 & 2.0 & 1.5 & 6.1 & NO, & 33 & 35.5 & 5.5 & 4,5 & 1.7 & 1.6 & 20 \\
\hline $10^{5}$ & $27.8^{\circ}$ & 66.5 & 7.9 & 2.5 & 1.1 & ND & $\mathrm{ND}$ & ND & 13.9 & 5.6 & 5.0 & 2.3 & ND & 61 \\
\hline 96 & 19.9 & 48.8 & 5.7 & 1.9 & 1.1 & ND & ND & ND & 70.5 & 4.0 & 3.6 & 1.0 & ND & 17 \\
\hline $8^{6}$ & 21.6 & 47.9 & 6.2 & 1.8 & 0.9 & 3.7 & ND & 37 & 64.1 & 4.9 & 4.5 & 1.2 & ND & 15,16 \\
\hline 7 & 24.1 & 50.9 & 6.4 & 2.0 & 1.0 & 5.0 & ND & 35 & 15.9 & 4.9 & 4.0 & 1.3 & 0.8 & 12,13 \\
\hline$\dot{0}$ & 23.2 & 57.2 & 7.1 & 2.2 & 1.0 & NU & 0.60 & 39 & 34.9 & 5.5 & 4.1 & 1.4 & ND & 10,11 \\
\hline 5 & 20.8 & 42.0 & 6.3 & 1.9 & ND & ND & ND & ND & 82.5 & 3.7 & 2.5 & 0.9 & ND & 9 \\
\hline 4 & 19.5 & 44.5 & 5.6 & 1.8 & 1.0 & 3.9 & ND & 41 & 114.0 & 4.3 & 3.6 & 1.1 & NU & 8 \\
\hline 3 & 22.3 & 53.7 & 5.8 & 2.1 & 1.0 & 4.7 & ND & 37 & 78.3 & 4.6 & 4.1 & 1.4 & ND & 3,4 \\
\hline 2 & 27.0 & 54.3 & 6.6 & 2.1 & 1.1 & ND & ND & ND & 45.8 & 4.9 & 2.3 & 1.6 & ND & 2 \\
\hline 1 & 24.1 & 49.6 & 6.5 & 2.0 & 1.7 & 8.5 & ND & 33 & 33.6 & 5.7 & 2.8 & 1.6 & ND & 1 \\
\hline Dike ${ }^{7}$ & ND & 61.9 & ND & 1.9 & 1.5 & ND & ND & ND & 30.8 & 4.9 & 5.0 & 1.6 & ND & 5 \\
\hline $\begin{array}{l}\text { Aver } \\
\text { and } \\
{ }^{2} \text { See } \\
{ }^{3} \text { Unio } \\
\text { Basa } \\
{ }^{4} \text { Meye } \\
{ }^{5} \text { Cent } \\
{ }^{6} \text { Roge } \\
{ }^{7} \text { Dike } \\
{ }^{7} D^{2}\end{array}$ & $\begin{array}{l}\text { igure } \\
\text { town-3 } \\
\text { t flow } \\
\text { Ridge } \\
\text { r Cree } \\
\text { sburg }\end{array}$ & $\begin{array}{l}4 \text { tor } \\
3 \text { flow } \\
\text { w. } \\
\text { e flow } \\
\text { ek flor } \\
\text { flows }\end{array}$ & E & (1) & 78) & do & Ide & $|t|$ & flow & & & & & \\
\hline
\end{tabular}


A study conducted by Price (1977) showed that at least 32 dikes (Figure 5) exposed within the vicinity of the type section are chemically similar to Grande Ronde Basalt. Chemical, field, petrographic, and paleomagnetic data further indicated that most of these dikes could have sourced the sequence of Group B Grande Ronde Basalt flows (Figure 4).

\section{PETROGRAPHY}

The Grande Ronde Basalt flows of the type section are generally fine grained and aphyric. Hand specimen samples are generally blue-black in color and contain occasional plagioclase phenocrysts and plagioclase-clinopyroxene glomerocrysts. The only notable exceptions are the two Rogersburg flows (Figure 4 ) which contain approximately 15 percent plagioclase phenocrysts averaging $1 / 2$ centimeter in length (Figure 6). The only other phyric flow in the section is the single Saddle Mountains Basalt flow, the Slippery Creek, which contains approximately 10 percent olivine phenocrysts averaging $1 / 4$ centimeter in size. 01 ivine phenocrysts are rare within the underlying Grande Ronde Basalt flows of the section.

In thin section, the Grande Ronde Basalt flows of the type locality generally display a hypocrystalline-intersertal or intergranular texture (Figure 6). The most common microphenocryst minerals include plagioclase $\left(A n_{40-60}\right)$ and augite. The most common groundmass minerals (1isted in general order of abundance) include plagioclase, augite, ilmenite and titanomagnetite, pigeonite and olivine, and orthopyroxene (see Reidel, 1978, for orthopyroxene discussion). Microphenocrysts and microlites are generally set in a matrix of tachylitic glass. Average electron microprobe analyses for minerals and intersertal glass constituents of selected Grande Ronde Basalt flows are listed in Table IV. Modal analyses and petrographic descriptions of flows of the section, as determined by Camp (1976), are listed in Tables V and VI, respectively. Camp (1976) found that, in general, Group A flows contain more olivine and less pigeonite than do Group B flows. Camp (1976) also concluded that the texture can vary with intraflow sample location and that individual Grande Ronde Basalt flows within the section cannot be distinguished on the basis of modal analyses. 


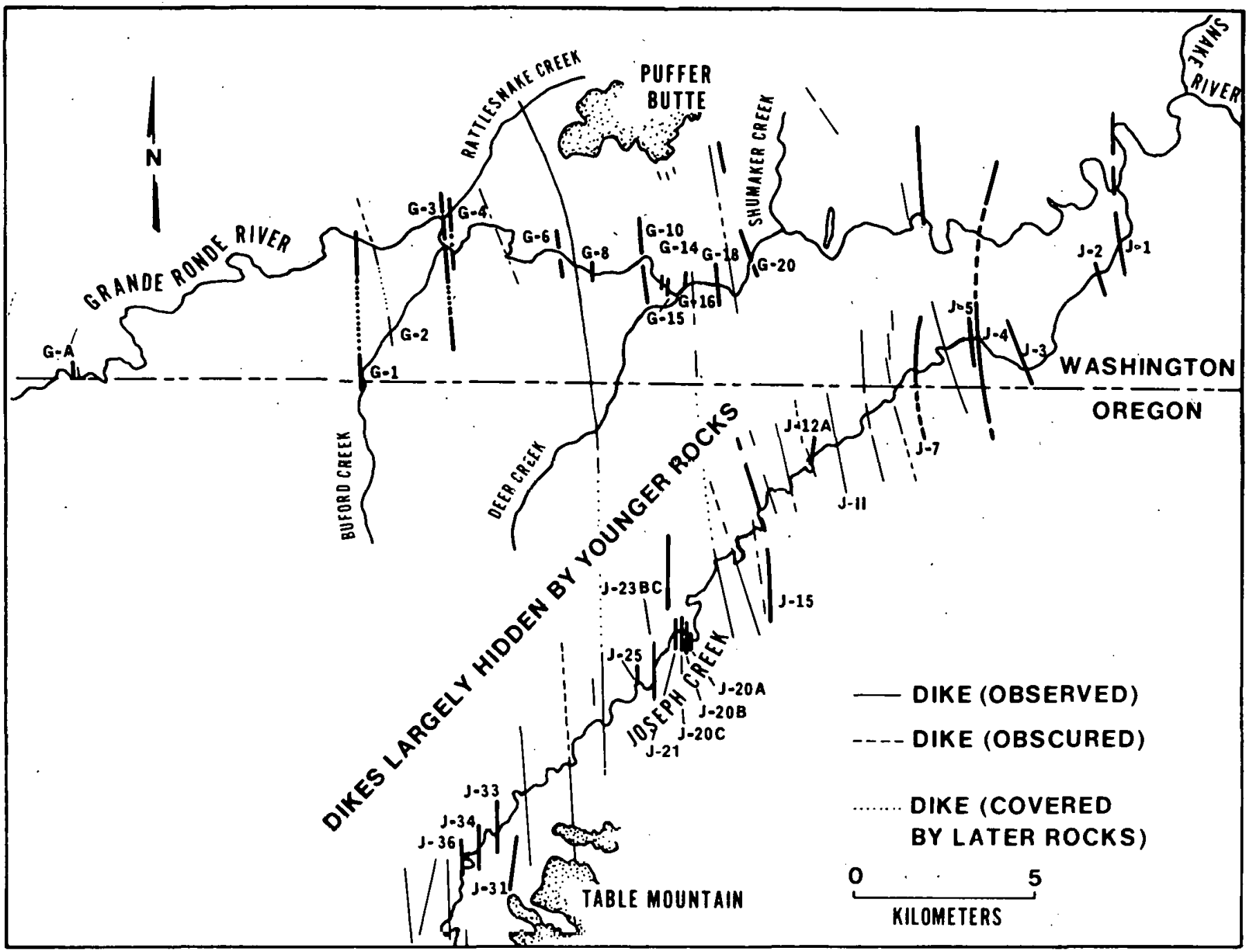

FIGURE 5

LOCATION OF COLUMBIA RIVER BASALT DIKES OF THE GRANDE RONDE CHEMICAL TYPE IN SOUTHEASTERN WASHINGTON AND NORTHEASTERN OREGON

(Grande Ronde Basalt dikes identified by number.) 


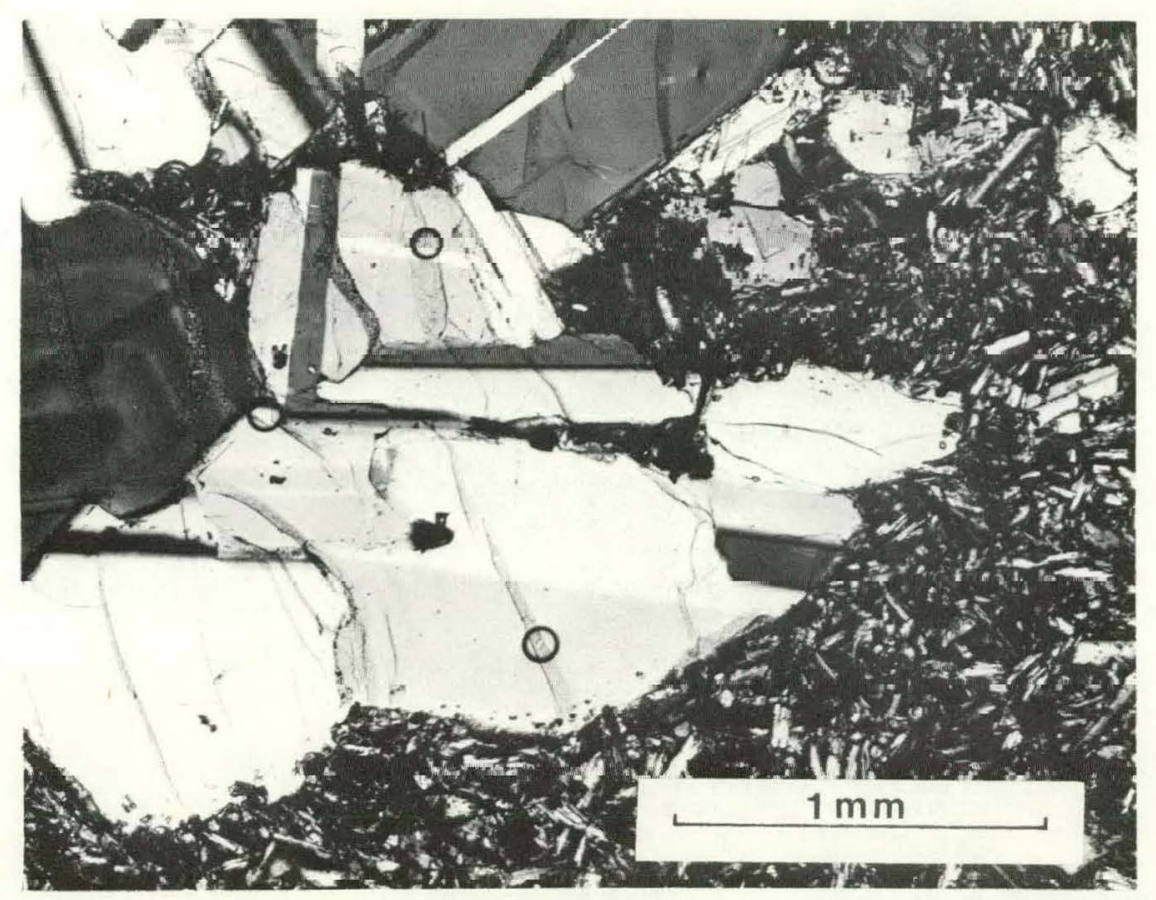

ROGERSBURG FLOW

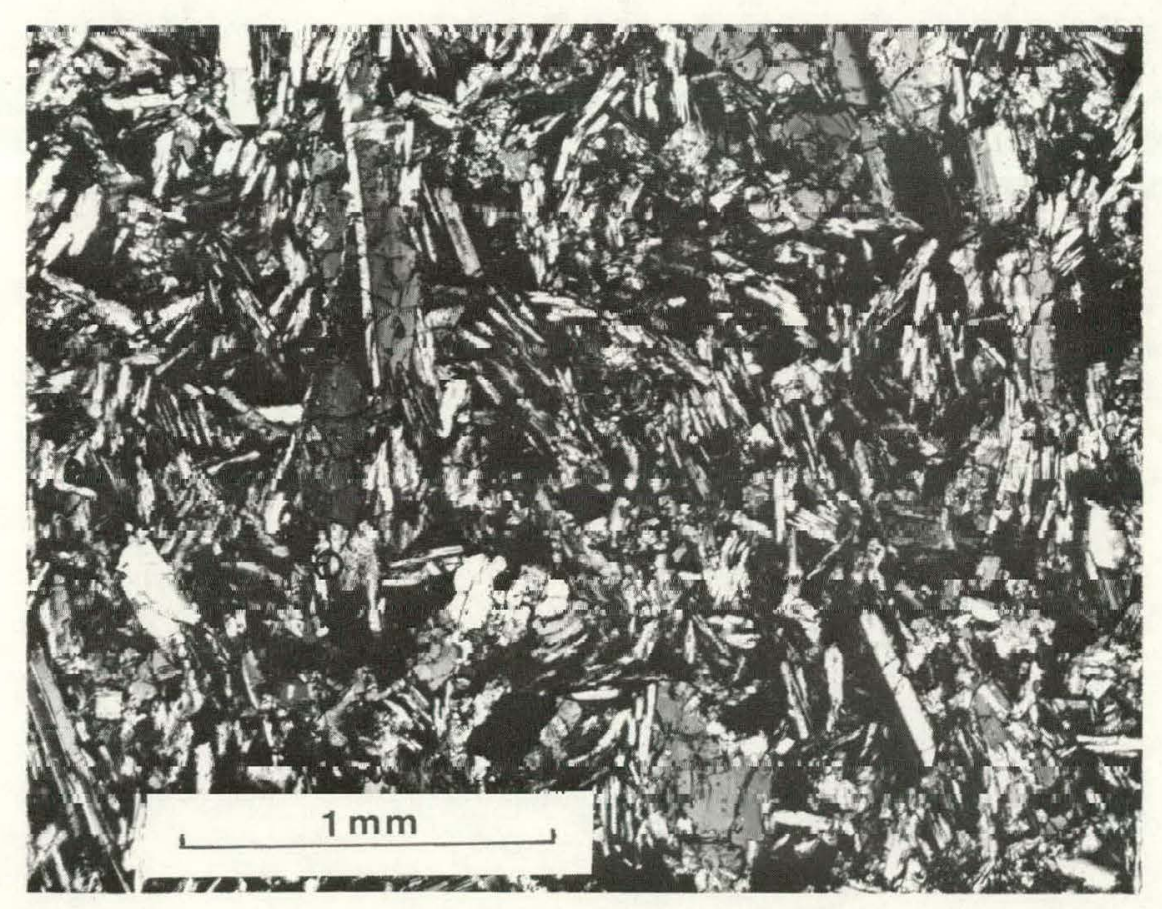

APHYRIC GRANDE RONDE BASALT FLOW

FIGURE 6

PHOTOMICROGRAPH OF ROGERSBURG FLOW AND TYPICAL APHYRIC GRANDE RONDE BASALT FLOW 


\section{TABLE IV}

MINERAL AND GLASS AVERAGE ELECTRON MICROPROEE ANALYSES FOR FLOWS OF THE GRANDE RONDE BASALT TYPE SECTIONI

\begin{tabular}{|c|c|c|c|c|c|c|c|}
\hline $\begin{array}{l}\text { Const ituent: } \\
\text { Flow No: }\end{array}$ & $\left(1,3, \frac{\text { Plagioclase }}{7,8,26,30,32)}\right.$ & $\frac{\text { Clinonyroxene }}{\frac{\text { Augite }}{T, 3,7,8,26,30,32)}}$ & $\frac{\text { Pigeonite }}{(8,30)}$ & $\frac{\text { Ilmenite }}{(3)}$ & $\left(1, \frac{\text { Titanomagnetite }}{3,7,8,30,31,} 32\right)$ & $\frac{01 \text { ivine }}{(7)}$ & $\frac{\text { Glass }}{(1,3,7,8,30,31,32)}$ \\
\hline \multicolumn{8}{|l|}{ (\%) } \\
\hline $\mathrm{SiO}_{2}$ & 55.2 & 53.5 & 53.8 & 0.70 & 1.0 & 38.2 & 74.2 \\
\hline $\mathrm{Al}_{2} \mathrm{O}_{3}$ & 27.2 & 2.3 & 0.20 & 0.50 & 1.8 & & 13.9 \\
\hline $\mathrm{FeO}^{\circ}$ & 0.70 & 13.5 & 21.8 & 46.5 & 55.2 & 44.7 & 1.5 \\
\hline $\mathrm{MgC}$ & & 13.3 & 18.0 & 1.3 & 1.1 & 16.2 & 2.7 \\
\hline $\mathrm{CaO}$ & 10.7 & 16.7 & 4.8 & 0.20 & 0.20 & 0.30 & 1.1 \\
\hline $\mathrm{Na}_{2} \mathrm{O}$ & 5.4 & & & & & & \\
\hline $\mathrm{K}_{2} \mathrm{C}$ & 0.50 & & & & & & 6.8 \\
\hline $\mathrm{MnC}$ & & & & 0.20 & 0.40 & 0.60 & \\
\hline $\mathrm{TiC}_{2}$ & & 0.20 & 0.30 & 50.6 & 30.4 & & 0.30 \\
\hline$P_{2} C_{5}$ & & 0.70 & 0.30 & & & & 0.20 \\
\hline Mole & $\mathrm{An}_{52} \mathrm{Ab}_{48}$ & $\mathrm{En}_{40} \mathrm{Fs}_{26} \cdot{ }^{10}{ }_{35}$ & $\mathrm{En}_{46}{ }^{\mathrm{Fs}} 44^{\mathrm{Wo}} 10$ & & & $\mathrm{Fo}_{37} \mathrm{Fa}_{63}$ & \\
\hline
\end{tabular}


TABLE $V$

MODAL ANALYSES FOR SAMPLES FROM THE GRANDE RONDE BASALT TYPE SECTION ${ }^{1}$

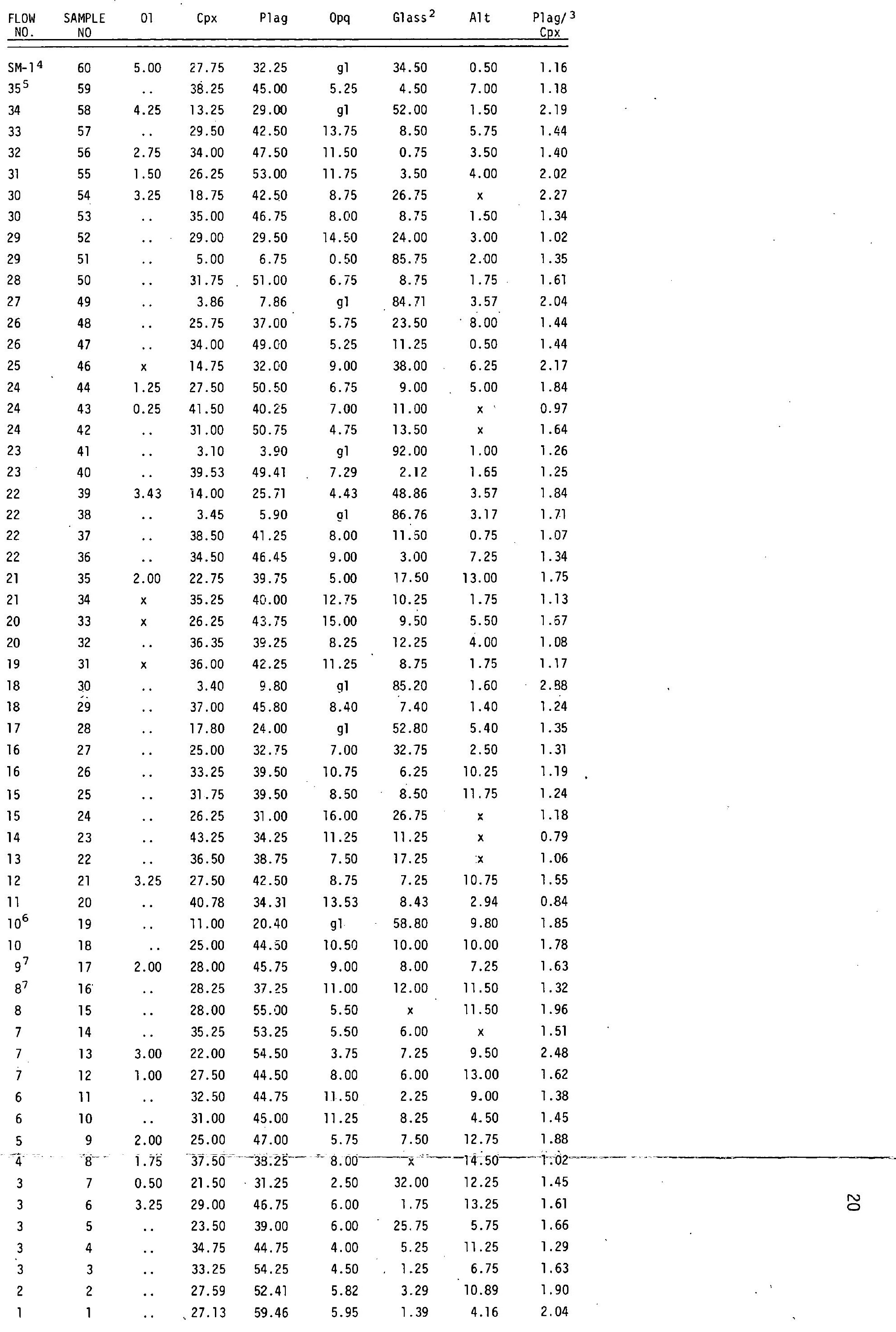

1 Modal analyses as reported in Camp (1966); $01=01$ ivine; $C p x=$ clinopyroxene;

Plag = plagioclase; $g l=$ percent included in glass content; $x=$ present in

small amounts; $A 1 t=$ alteration.

2 Glass value may include cryptocrystalline material and microlites.

$3 \mathrm{Plag} / \mathrm{Cpx}=$ plagioclase-clinopyroxene ratio.

4 Uniontown-3 flow of Camp (1966), Slippery Creek flow of Price (1977); Saddle Mountains Basalt flow.

5 Meyer Ridge flow of Camp (1976); Grande Ronde Basalt flow.

6 Center Creek flow of Reidel (1978).

7 Rogersburg flows of Price (1977) and porphyritic flows of Camp (1976) and

Reidel (1978). 
TABLE VI

PETROGRAPHY OF BASALT SAMPLES FROM THE GRANDE RONDE BASALT TYPE SECTION

\begin{tabular}{|c|c|c|c|c|c|c|c|c|c|}
\hline $\begin{array}{l}\text { Flow } \\
\text { Number }\end{array}$ & $\begin{array}{l}\text { Sample } \\
\text { Number }\end{array}$ & $\begin{array}{c}\text { Matrix } \\
\text { Grain Size* }\end{array}$ & Plag. & 01 & $\mathrm{Cpx}$ & $C \cdot 3 x\left(2 v 8 \pm 2^{\circ}\right)$ & $\begin{array}{c}\text { Opaque } \\
\text { Form }\end{array}$ & Glass & $\begin{array}{l}\text { Remarks, } \\
\text { Texture }\end{array}$ \\
\hline$S M-1^{3}$ & GR 60 & $0.10-0.30$ & $\mathrm{~g}$ & a & $\mathrm{mp}$ & $47.0,48.0,48.0,48.0$ & $\begin{array}{l}\text { equant to } \\
\text { elongate }\end{array}$ & opaque & hya loophitic \\
\hline $35^{4}$ & GR 59 & $0.10-0.50$ & $g$ & .. & 9 & $47.0,43.0-48.5^{\dagger}, 43.5-5.0^{\dagger}$ & $\begin{array}{l}\text { equant to } \\
\text { elongate }\end{array}$ & pale & intergranular \\
\hline 34 & GR 58 & $0.10-0.30$ & $g$ & a & $g$ & $47.0,47.0,48.0,48.0$ & (?) & $r \geqslant d$ & hyaloophitic \\
\hline 33 & GR 57 & $0.05-0.15$ & phen & $\cdot \cdot$ & $g$ & $46.0-19.0^{+}, 47.0$ & equant & pale & intergranular \\
\hline 32 & GR 56 & $0.10-0.50$ & 9 & $g$ & $g$ & $46.0-21.0^{\dagger}, 40.0,46.0$ & equant & pale & intergranular \\
\hline 31 & GR 55 & $0.10-0.40$ & 9 & 9 & $\mathrm{mp}$ & 47.0 & equant & brown & intergranular \\
\hline 30 & GR 54 & $0.10-0.25$ & phen & a & $\mathrm{g}$ & & $\begin{array}{l}\text { equant to } \\
\text { elongate }\end{array}$ & pale & intersertal \\
\hline 30 & GR 53 & $0.10-0.30$ & $g$ & $\cdot$. & mp & $39.0-15.0^{+}, 41.0$ & equant & brown & intergranular \\
\hline 29 & GR 52 & $0.05-0.20$ & mp & .. & mp & 46.0 & equant & brown & intergranular \\
\hline 29 & GR 51 & $0.05-0.20$ & $m p$ & $\cdot$. & 9 & $51.5-40.0^{\dagger}, 48.0-33.0^{+}, 45.0$ & equant & opaque & pilotaxitic \\
\hline 28 & GR 50 & $0.10-0.30$ & $\mathrm{mp}$ & & mp & $43.5,13.0$ & equant & pale & intergranular \\
\hline 27 & GR 49 & $0.05-0.15$ & $\mathrm{mp}$ & $\cdot$. & $\mathrm{mp}$ & $33.0-8.5^{4}, 42.0$ & equant & brown & intergranular \\
\hline 26 & GR 48 & $0.10-0.30$ & $\mathrm{mp}$ & .. & $\mathrm{mp}$ & 40.0 & equant & brown & intergranular \\
\hline 26 & GR 47 & $0.10-0.50$ & 9 & $\cdot \cdot$ & 9 & 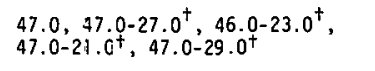 & equant & brown & intergranular \\
\hline 25 & GR 46 & $0.10-0.30$ & mp & $\mathrm{g}$ & $\mathrm{g}$ & 47.0 & $\begin{array}{l}\text { equant to } \\
\text { elongate }\end{array}$ & brown & intersertal \\
\hline 24 & GR 44 & $0.10-0.40$ & $\mathrm{mp}$ & $\mathrm{s}$ & 9 & $46.0-35.0^{\dagger}, 48.0,40.0,47.0$ & $\begin{array}{l}\text { equant to } \\
\text { elongate }\end{array}$ & pale & intergranular \\
\hline 24 & GR 43 & $0.10-0.40$ & $\mathrm{mp}$ & $\mathrm{s}$ & mp & $\begin{array}{l}48.0-33 . .^{\dagger}, 50.0-34.0^{\dagger}, 50.0- \\
35.0^{\dagger}, 46.0,49.5\end{array}$ & $\begin{array}{l}\text { equant to } \\
\text { elongate }\end{array}$ & brown & intergranular \\
\hline 24 & GR 42 & $0.10-0.40$ & 9 & . & 9 & 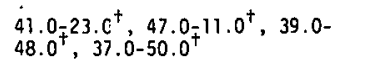 & equant & brown & intergranular \\
\hline 23 & GR 41 & $0.03-0.15$ & 9 & ". & 9 & $40.0-14 . c^{+}, 41.0-21.0^{\dagger}, 41.0$ & equant & brown & variolitic \\
\hline 23 & GR 40 & $0.03-0.20$ & mp & $\cdot \cdot$ & $g$ & $41.0-13 . \varepsilon^{\dagger}, 42.0$ & equant & brown & intergranular \\
\hline 22 & GR 39 & $0.03-0.15$ & $g$ & a & $g$ & $45.0,36.0-8.0^{\dagger}, 45.0$ & equant & red & intersertal \\
\hline 22 & GR 38 & $0.03-0.15$ & $\mathrm{mp}$ & $\cdot \cdot$ & mp & $49.5,48.0,43.0,41.0$ & equant & brown & pilotaxitic \\
\hline 22 & GR 37 & $0 . c 5-0.20$ & $g$ & $\cdot \cdot$ & 9 & $47.0-35 . c^{\dagger}, 43.0-13.5^{\dagger}, 14.5$ & equant & brown & intergranular \\
\hline 22 & GR 36 & $0.65-0.20$ & 9 & $\cdot \cdot$ & 9 & $43.0-10 . c^{\dagger}, 40.0-15.5^{\dagger}$ & equant & pale & intergranular \\
\hline 21 & GR 35 & $0.10-0.40$ & $g$ & 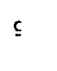 & 9 & 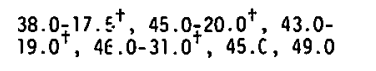 & equant & brown & intersertal \\
\hline 21 & GR 34 & $0.10-0.40$ & 9 & $\varsigma$ & 9 & $38.0-57 . c^{\dagger}, 43.0,46.0,46.0$ & equant & brown & intergranular \\
\hline 20 & GR 33 & $0.10-0.40$ & $g$ & $\varsigma$ & $\mathrm{mp} \cdot$ & $\begin{array}{l}40.0-13 . ._{5}^{t}, 43.0,43.0,48.0, \\
40.0-43 . E^{+}\end{array}$ & equant & brown & intergranular \\
\hline 20 & GR 32 & $0.05-0.20$ & 9 & $\cdot \cdot$ & $g$ & $42.0-43.6^{\dagger}, 22.0-6.0^{\dagger}$ & equant & brown & intergranular \\
\hline 19 & GR 31 & $0.10-0.40$ & $g$ & $\varsigma$ & 9 & $\begin{array}{l}48.0-41.60^{\dagger}, 44.0-49.5^{\dagger}, 45.0 \\
43.0,40.0\end{array}$ & equant & brown & intergranular \\
\hline 18 & GR 30 & $0.03-0.10$ & 9 & $\cdot \cdot$ & 9 & & equant & brown & pilotaxitic \\
\hline 18 & GR 29 & $0.05-0.25$ & 9 & .. & g & & equant & brown & intergranular \\
\hline 17 & GR 28 & $0.05-0.15$ & mp & •. & 9 & $\begin{array}{l}42.0-49.5^{+}, 35.5-48.0^{\dagger}, 43.0 \\
\end{array}$ & (?) & opaque & hyaloophitic \\
\hline 16 & GR 27 & $0.10-0.25$ & $\mathrm{~g}$ & ". & $g$ & 41.0 & equant & brown & intergranular \\
\hline 16 & GR 26 & $0.10-0.40$ & $g$ & $\cdot$. & $g$ & $43.0,42.0-10.0^{+}$ & equant & pale & intergranular \\
\hline 15 & GR 25 & $0.0 .15-0.20$ & $g$ & $\cdot$. & mp & $40.0,40.0,32.0-46.0^{\dagger}$ & equant & pale & intergranular \\
\hline 15 & GR 24 & $0.03-0.15$ & mp & . & $\mathrm{g}$ & $\begin{array}{l}38.00^{1} 13.5^{+}, 47.0-032.0^{\dagger}, 46.0- \\
29.0^{\dagger}, 44.0-30.0^{3}\end{array}$ & equant & brown & intergranular \\
\hline 14 & GR 23 & $0.65-0.20$ & 9 & ". & $\mathrm{g}$ & & equant & brown & intergranular \\
\hline 13 & GR 22 & $0.67-0.30$ & 9 & $\cdot \cdot$ & $g$ & $34.5-12.6^{\dagger}, 34.5-41.5^{\dagger}, 40.0$ & equant & pale & hyaloophitic \\
\hline 12 & GR 21 & $0.10-0.30$ & $g$ & a & $g$ & $49.5,48.0$ & $\begin{array}{l}\text { equant to } \\
\text { elongate }\end{array}$ & brown & intergranular \\
\hline 11 & GR 20 & $0 .: 0-0.25$ & g & .. & 9 & & 巳quant & t:rown & intergranular \\
\hline $10^{5}$ & GR. 19 & $0.05-0.15$ & phen &.$\cdot$ & 9 & $46.5,48.0$ & equant & trown & intersertal \\
\hline 10 & GR 18 & $0.30-0.30$ & phen & .. & 9 & $46.0,46.0$ & equant & trown & intergranular \\
\hline $9^{6}$ & GR 17 & $0.05-c .20$ & mp & 9 & 9 & $43.0,43.0,42.0$ & equant & trown & intergranular \\
\hline $8^{6}$ & GR 16 & $0.03-c .10$ & phen & & 9 & $34.0-10.0^{+}, 45.0,46.0,40.0$ & Equant & cpaque & intergranular \\
\hline 8 & GR 15 & $0.05-c .20$ & phen & $\cdot \cdot$ & g & $\begin{array}{l}34.0-14.5^{\dagger}, 43.5,42.0,47.0 \\
42.0\end{array}$ & $\begin{array}{l}\text { Equant to } \\
\text { elongate }\end{array}$ & .. & intergranular \\
\hline 7 & GR 14 & $0.10-C .30$ & $\mathrm{~g}$ & •. & $\mathrm{g}$ & $42.0,45.0,39,0,39.0$ & equant & trown & intergranular \\
\hline 7 & GR 13 & $0.10-c .30$ & phen & a & 9 & $47.0,43.5,41.0$ & equant & trown & $\begin{array}{l}\text { intergranular, } \\
\text { porphyritic }\end{array}$ \\
\hline 7 & GR 12 & $0.10-c .30$ & phen & 9 & $\mathrm{~g}$ & 43.0 & equant & brown & $\begin{array}{l}\text { intergranular, } \\
\text { porphyritic }\end{array}$ \\
\hline $6^{-\cdots}$ & GR 71 & $-0005=€: 20^{-}$ & $\mathrm{mp}^{-\cdots}$ & ...... & $g^{2}$ & 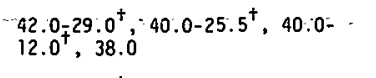 & -equant $=\ldots$ & brown- & intergranular- \\
\hline \multirow[t]{2}{*}{6} & GR 10 & $0.05-c .15$ & $\mathrm{mp}$ & .. & mp & $\begin{array}{l}31.0-11.5^{4}, 43.0,31.0,40.8 \\
39.0,42.0\end{array}$ & equant & trown & intergranular \\
\hline & GR 9 & $0.10-c .40$ & 9 & 9 & g & $48.5,48.5,48.0$ & $\begin{array}{l}\text { elongate } \\
\text { to equant }\end{array}$ & pale & intergranular \\
\hline 4 & GR 8 & $0.15-(1.25$ & 9 & 9 & $g$ & $46.0,42.0,48.5,48.0$ & longate & pale & intergranular \\
\hline 3 & GR 7 & $0.05-6.20$ & mp & 9 & 9 & $43.0,41.0,46.0,41.0$ & Elongate & opaque & intersertal \\
\hline 3 & GR 6 & $0.05-(1.20$ & mp & a & 9 & $45.0,46.0,47.0$ & elongate & pale & intergranular \\
\hline 3 & GR 5 & $0.15-0.50$ & 9 & $\cdot \cdot$ & $g$ & $41.0,43.5,41.0,41.0$ & equant & pale & hyaloophitic \\
\hline 3 & GR 4 & $0.05-(1.25$ & 9 & $\cdot \cdot$ & $g$ & $43.5,45.0,40.0,43.5$ & $\begin{array}{l}\text { equant to } \\
\text { equngate }\end{array}$ & trown & intergranular \\
\hline 3 & GR 3 & $0.05-0.25$ & $\mathrm{mp}$ & $\cdot \cdot$ & 9 & $42.0-17.0^{\dagger}, 43.0-18.0^{\dagger}$ & equant & pale & intergranular \\
\hline 2 & GR 2 & $0.05-0.25$ & $g$ & $\cdot \cdot$ & mp & $46.0,45.5$ & equant & pale & intergranular \\
\hline 1 & GR 1 & $0.10-0.40$ & 9 & $\cdot \cdot$ & 9 & $\begin{array}{l}43.0,46.0-23.0^{\dagger}, 46.0,20.0, \\
15.0\end{array}$ & equant & pale & intergranular \\
\hline
\end{tabular}

Petrographic descriptions as reported in Camp (1976); plag = Flagioclase; phen = phenocrysts; $01=01$ ivine; $C p x=c 1$ inopyroxene 2 see figure 4 for flow divisions and sample localities.

3 Uniontown-3 flow of Camp (1976), S1ippery Creek flow of Price (1977); Saddle Mountains Basalt flow.

${ }^{4}$ Meyer Ridge flow of Camp (1976); Grande Ronde Easalt flow.

${ }^{5}$ Center Creek flow' of Reidel (1978).

${ }^{6}$ Rogersburg flows of Price (1977) and porphyritic flows of Camp: (1976) and Reidel (1978).

A Average length of playioclase laths in matrix (millimeters).

$t_{\text {Zoned from margin to core. }}$ 


\section{APPLICATION OF SECTION DATA}

This document was compiled to provide a framework upon which more regional stratigraphic and petrogenetic studies of the Grande Ronde Basalt can be based. The Grande Ronde Basalt type section lies near the approximate center of an over 6,500-square-kilometer area of the Columbia Plateau in which flows and dikes of the Columbia River Basalt Group have recently been examined in detail by Camp (1976), Price (1977), Reidel (1978), and Ross (1978). As such, the Grande Ronde Basalt type section is only one of nearly 50 basalt sections which have been measured within this area. Paleomagnetic, petrologic, and chemical data are available for at least 25 of these sections. It is anticipated that integration and interpretation of these data will provide a better understanding of the relation between Grande Ronde Basalt flows and source dikes located near the eastern margin of the Columbia Plateau and Grande Ronde Basalt flows currently being esamined by Rockwell Hanford Operations within the Pasco Basin.

\section{ACKNOWLEDGMENTS}

Section research conducted by Drs. V. E. Camp (1976) and S. P. Reide 1 (1978) was partially supported by National Science Foundation Grant Number 39802 to Dr. P. R. Hooper of Washington State University. Research conducted by Dr. S. M. Price (1977) was partially sponsored by the U. S. Atomic Energy Commission, Contract No. AT(45-1)-2042, while on a Northwest College and University for Science Appointment. Gratitude is expressed to Drs. P. R. Hooper, W. D. Kleck, M. R. Ross, and Messrs. G. S. Holden and $W$. H. Price who assisted the authors in section measurement and sample collection.

\section{BIBL IOGRAPHY}

Bond, J. G., 1962, Geology of The Clearwater Embayment in Idaho, Ph. D. Thesis, University of Washington, Seattle, Washington, $193 \mathrm{p}$.

Breeser, P. J., 1972, General Geology and Highway Relignment Considerations in the Pinehurst-New Meadows-Tamerack Area, West-Central Idaho, M. S. thesis, University of Idaho, Moscow Idaho, $103 \mathrm{p}$. 
Camp, V. E., 1976, Petrochemical Stratigraphy and Structure of the Columbia River Basalt, Lewiston Basin Area, Idaho-Washington, Ph. D. Thesis, Washington State University, Pullman, Washington, $201 \mathrm{p}$.

Gibson, I. L., 1969 "A Comparative Account of Flood Basalt Volcanism of the Columbia Plateau and Eastern Iceland," Bullet in of Volcanology, 33, p. 420-437.

Hooper, P. R., Reidel, S. P., Brown, J. C., Bush, J. H., Holden, G. S., Kleck, W. D., Robinette, M., Sundstrom, C. E., and Taylor, T. L., 1976, "Major Element Analyses of Columbia River Basalt, Part I, "Basalt" Research Group, Washington State University Open-file Report.

Price, S. M., 1977, An Evaluation of Dike-Flow Correlations Indicated by Geochemistry, Chief Joseph Swarm, Columbia River Basalt, Ph. D. Thesis, University of Idaho, Moscow, Idaho, $320 \mathrm{p}$.

Reide1, S. P., 1978, The Stratigraphy and Petrogenes is of the Grande Ronde Basalt in the Lower Salmon and Adjacent Snake River Canyons, Ph. 0 Thesis, Washington State University, Pul1man, Washington, $415 \mathrm{p}$.

Ross, M. E., 1978, Stratigraphy, Structure, and Petrology of Columbia River Basalt in a Portion of the Grande Ronde River - Blue Mountains Area of Oregon and Washington, Ph. D. Thesis, University of Idaho, Moscow, Idaho, $413 \mathrm{p}$.

Swanson, D. A. and Wright, T. L., 1976, "Magnetostratigraphic Units In. the Yakima Basalt, Southeast Washington," Geological Society of America, Abstracts With Programs (Cordilleran Section), 8 (3), p. 413 .

Swanson, D. A. and, Wright, T. L., 1978, "Bedrock Geology of The Northern Columbia Plateau and Adjacent Areas," in Baker, V. R., and Nummedal, D. editors, The Channeled Scabland, National Aeronautics and Space Adininistration, Washington U. C., p. 37-57.

Swanson, D. A., Wright, T. L., Hooper, P. R., and Bentley, R. D., 1978 (in press), "Revisions in Stratigraphic Nomenclature of The Columbia River Basalt Group, Contributions to Stratigraphy," U. S. Geological Survey Bullet in 1457-H, .

Taubeneck, W. H., 1970, "Dikes Of The Columbia River Basalt In Northeastern Oregon, Western Idaho, and Southeastern Washington," in Proceedings, Second Columbia River Basalt Symposium, Cheney, Washington, 1969, Eastern Washington State College Press, Cheney, Washington, $\mathrm{p}$. $\overline{73-96 .}$

Waters, A. C., 1961, "Stratigraphy and Lithologic Variations in the Columbia River Basalt," American Journal of Science, 259, p. 583-611

Wright, 1. L., Grolier, M. J., and Swanson, D. A., 1973, "Chemical Variation Related to the Stratigraphy of the Columbia River Basalt," Geological Society of America Bulletin, 84, p. 371-386. 


\section{DISTR IBUTION}

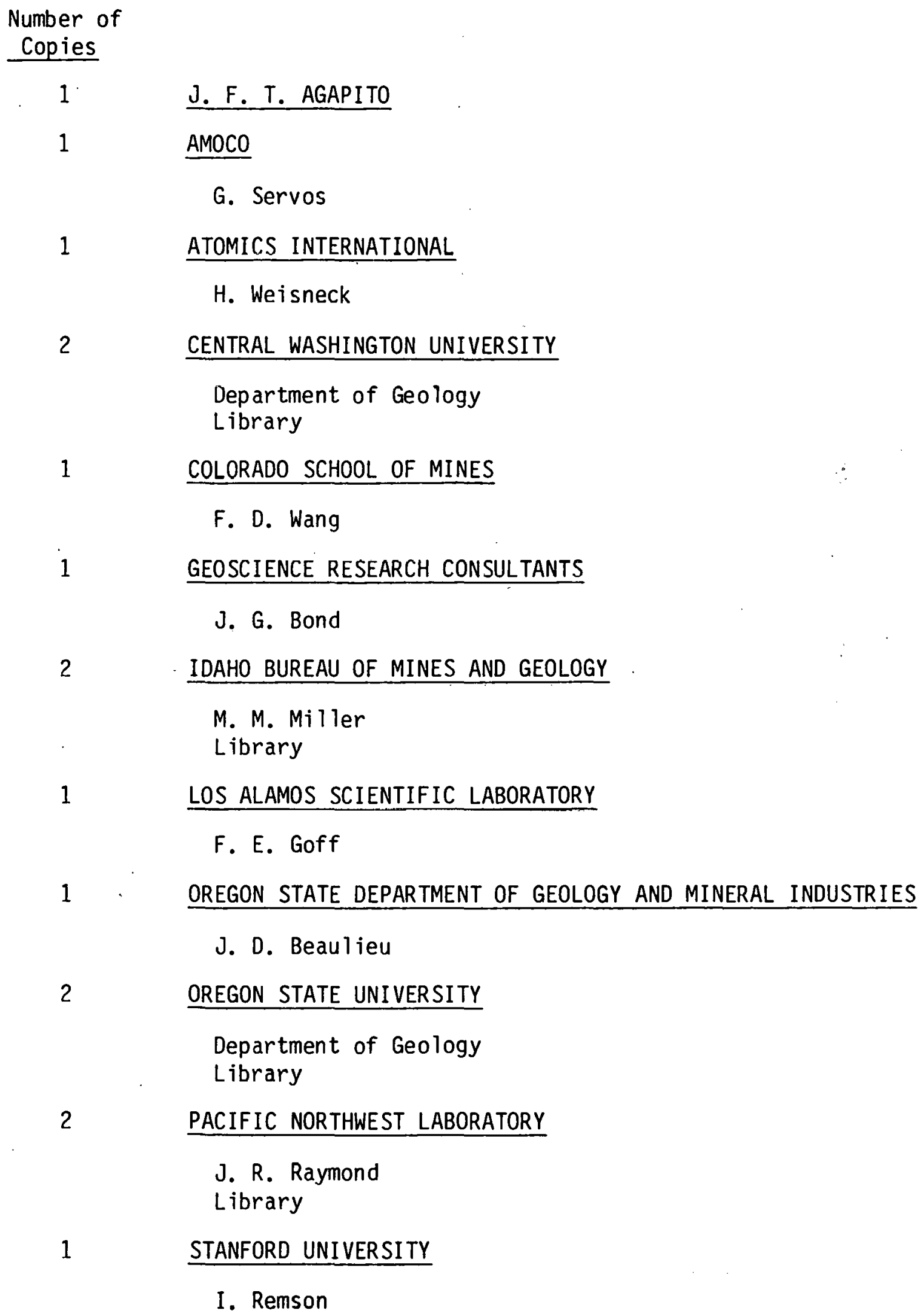


Number of

Copies

1

U. S. ARMY-YAKIMA FIRING CENTER

W. R. Dietderich

1

U. S. BUREAU OF MINES

J. W. Corwine

1

U. S. BUREAU OF RECLAMATION

Columbia Bas in Project Geologist

1

U. S. DEPARTMENT OF ENERGY-COLUMBUS PROGRAM OFFICE

J. Neff

2

U. S. DEPARTMENT OF ENERGY-HEADQUARTERS

M. W. Frei

D. L. Veith

3

U. S. DEPARTMENT OF ENERGY-RICHLAND OPERATIONS OFFICE

0. J. Elgert

R. B. Goranson

D. J. Squires

3

U. S. GEOLOGICAL SURVEY

C. Collier

R. Schneider

U. A. Swanson

2

UNIVERSITY OF IDAHO

Department of Geology

Library

2

UNIVERSITY OF OREGON

Department of Geology

Library

4

UNIVERSITY OF WASHINGTON

S. D. Malone

S. W. Smith

Department of Geology

Library 
Number of

Copies

1

1

2

2

2

1

2
VANDERBILT UNIVERSITY

F. L. Parker

WASHINGTON PUBLIC POWER SUPPLY SYSTEM, INC.

D. D. Tillson

WASHINGTON STATE DEPARTMENT OF ECOLOGY

P. M. Grimstad

Library

WASHINGTON STATE DEPARTMENT OF NATURAL RESOURCES

V. E. Livingston

Library

WASHINGTON STATE UNIVERSITY

Department of Geology

Library

A. C. WATERS

WESTERN WASHINGTON UNIVER SITY

Department of Geology

Library

ROCKWELL HANFORD OPERATIONS

H. Babad

D. J. Brown

D. J. Cockeram

T. A. Curran (5)

R. A. Deju (2)

R. C. Edwards

R. E. Gephart

R. J. Gimera

R. E. Isaacson

C. W. Myers

W. H. Price (5)

M. J. Smith

Basalt Waste Isolation Program Library (15) Document Control (4) 
Form ĖRDA 426
$(2 \pi 5)$

ERDAM 3201

\section{U.S. ENERGY RESEARCH \& DEVELOPMIENT ADMINISTRATION \\ MAJOR CONTRACTOR'S RECOMMENDATION FOR \\ DISPOSITION OF SCIENTIFIC AND TECHNICAL DOCUMENT}

* See Instructions on Reverse

1. ERDA Report No RHO-BWI-LD-15

2. Subject Category No. UC-11

4. Type of Document (" $x$ " one)

a. Scientific and Technical Report

$\square$ b. Conference paper:

Title of conference

Date of conference

Exact location of conference

c. Other (Specify, Thesis, Translation, etc.)*

5. Copies Transmitted ("X" one or more)

$\square$ a. Copies being transmitted for standard distribution by ERDA-TIC.

$\square$ b. Copies being transritted for special distribution per attached complete address list.*

[X c. Two completely legible, reproducible copies being transmitted to ERDA-TIC.

6. Recommended Distribution (" $X$ " one)

A. Normal handling (after Patent clearance): no restraints ondistribution except as may be required by the security classification.

$\square$ b. Make available only to U.S. Government agencies and their contractors.

$\square$ c. Make available only within ERDA and to ERDA contractors.

$\square$ d. Make available only within ERDA.

$\square$ e. Make available only to those listed in item 12 below.

$\square$ f. Other (Specify)*

7. Recommended Announcement (" $x$ " one)

a. Normal proccdure may be followed.*

$\square$ b. Recommend following announcement limitations:

8. Reason for Restrictions Recommended in 6 or 7 above.

- $\square$ a. Preliminary information.

$\square$ b. Prepared primarily for internal use.

$\square$ c. Other (Explain)

9. Patent Clearance (" $X$ " one)

X. ERDA patent clearance has been granted by responsible ERDA pat ent group.

$\square$ b. Document has been sent to responsible ERDA patent group for clearance.

10. National Security Information (For classified document only; " $X$ " one)

$\square$ a. Document does contain national security Information other than restricted data.

b. Document does not contain national security information otner than itestricted data.

11. Copy Reproduction and Distribution

a. Total number of copies reproduced -100

b. Number of copies distributed outside originating organization 36 INCLUDING 2 TO IIC

12. Additional Information or Remarks (Continue on separate sheet, if necessary)

13. Submitted by (Name and Position) (Please print or type)*

D. C. LEYSON, MANAGER, RECORDS MANAGEMENT

14. Organization

ROCKWELL HANFORD OPERATIONS, RICHLAND, WA. 99352

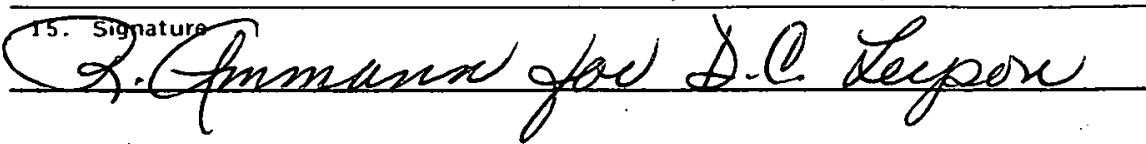

16. Date 